\title{
Monoplane 3D Overlay Roadmap versus Conventional Biplane 2D Roadmap Technique for Neurointervenional Procedures
}

\author{
Dong-Kyu Jang, MD, PhD', David A. Stidd, MD, MS', Sebastian Schafer, $\mathrm{PhD}^{3}$, \\ Michael Chen, MD², Roham Moftakhar, MD², Demetrius K. Lopes, MD²
}

Purpose: We investigated whether a 3D overlay roadmap using monoplane fluoroscopy offers advantages over a conventional 2D roadmap using biplane fluoroscopy during endovascular aneurysm treatment.

Materials and Methods: A retrospective chart review was conducted for 131 consecutive cerebral aneurysm embolizations by three neurointerventionalists at a single institution. Allowing for a transition period, the periods from January 2012 to August 2012 (Time Period 1) and February 2013 to July 2013 (Time Period 2) were analyzed for radiation exposure, contrast administration, fluoroscopy time, procedure time, angiographic results, and perioperative complications. Two neurointerventionalists (Group 1) used a conventional 2D roadmap for both Time Periods, and one neurointerventionalist (Group 2) transitioned from a 2D roadmap during Time Period 1 to a 3D overlay roadmap during Time Period 2.

Results: During Time Period 2, Group 2 demonstrated reduced fluoroscopy time $(p<0.001)$, procedure time $(P=0.023)$, total radiation dose $(p=0.001)$, and fluoroscopy dose $(P=0.017)$ relative to Group 1 . During Time Period 2, there was no difference of immediate angiographic results and procedure complications between the two groups. Through the transition from Time Period 1 to Time Period 2 , Group 2 demonstrated decreased fluoroscopy time $(p<0.001)$, procedure time $(p=0.022)$, and procedure complication rate $(p=0.041)$ in Time Period 2 relative to Time Period 1.

Conclusion: The monoplane 3D overlay roadmap technique reduced fluoroscopy dose and fluoroscopy time during neurointervention of cerebral aneurysms with similar angiographic occlusions and complications rate relative to biplane 2D roadmap, which implies possible compensation of limitations of monoplane fluoroscopy by 3D overlay technique.

Key Words : 3D roadmap; Aneurysms; Neurointervention; Fluoroscopy dose; Fluoroscopy time

\footnotetext{
'Department of Neurosurgery, Incheon St. Mary's Hospital, College of Medicine, The Catholic University of Korea, Incheon, Korea ${ }^{2}$ Department of Neurosurgery, Rush University Medical Center, Chicago, IL, USA

${ }^{3}$ Siemens Medical Solutions, Hoffman Estates, IL, USA

Received July 21, 2016; accepted after revision August 17, 2016.

Correspondence to: Demetrius K. Lopes, MD, Department of Neurosurgery, Rush University Medical Center, 1725 W. Harrison Street, Suite 855, Chicago, Illinois 60612, USA

Tel. 312-563-3394 Fax. 312-942-2176 E-mail: brainaneurysm@mac.com

This is an Open Access article distributed under the terms of the Creative Commons Attribution Non-Commercial License (http://creativecommons.org/licenses/by-nc/3.0) which permits unrestricted non-commercial use, distribution, and reproduction in any medium, provided the original work is properly cited.
} 


\section{Dong-Kyu Jang, et al.}

Three-dimensional (3D) DSA has been widely used to elucidate complex vascular diseases and guide treatment management [1-4]. The detailed aneurysm morphology and anatomical relationship between aneurysm and parent artery provided by 3D DSA have improved patient outcome and decreased the perioperative complications during endovascular treatment $[1,5]$. Information obtained from 3D DSA data has been used for proper interventional case selection and is routinely used to determine working angle projections during procedures. However, the use of the $3 \mathrm{D}$ volume reconstruction as a $3 \mathrm{D}$ overlay roadmap during neuroendovascular interventions has only been the subject of a few clinical report $[3,6,7]$.

Since 3D DSA provides comprehensive geometric visualization of cerebral vessel anatomy and aneurysm morphology, we hypothesized that 3D overlay roadmap technology may reduce neurointervention procedure time, and in turn reduce radiation dose, fluoroscopy time, and contrast volume. In addition, biplane angiographic equipment has been regarded an essential tool to conduct neuroendovascular procedures to improve neurointerventionists' comprehension of the spatial relationship of devices and target vessels, and reduce radiation dose compared to monoplane angiography system [8]. These have urged the upfront hospital investment for a biplane angiography suite.

To date, there is no direct comparative study evaluating clinical efficacy using 3D overlay roadmap technique relative to $2 \mathrm{D}$ roadmap angiography during neurointerventional procedures. The aim of this current study is to investigate whether $3 \mathrm{D}$ overlay roadmap technique using monoplane fluoroscopy offers clinical advantages over conventional $2 \mathrm{D}$ roadmap technique using biplane fluoroscopy during coil embolization of intracranial aneurysms.

\section{MATERIALS AND METHODS}

\section{Study Design}

This study was approved by the Institutional Review Board. A retrospective chart review was performed to identify coil embolization procedures for cerebral aneurysms performed by three neurointerventionalists (Demetrius K. Lopes, Michael Chen, Roham Moftakhar) from January 2012 through August 2013 at a single tertiary care center. Two neurointerventionalists (Group 1) used a conventional 2D roadmap technique throughout the review period, and the third neurointerventionalist (Group 2) changed from the conventional $2 \mathrm{D}$ roadmap technique to a $3 \mathrm{D}$ overlay roadmap technique half way through the study period. Allowing for a transition period to master the $3 \mathrm{D}$ roadmap technique, Time Period 1 was defined from January 2012 through August 2012 and Time Period 2 was defined from February 2013 through July 2013. All coil embolization procedures were conducted with the same biplane angiography system (Artis Zee, Siemens Healthcare, Forchheim, Germany) with volumetric reconstructions performed on a separate 3D workstation (syngo X Workplace, Siemens Healthcare, Forchheim, Germany). The two neurointerventionalists within Group 1 acquired and analyzed rotational 3D angiographic image data for procedure planning and working angle determination, and used conventional biplane 2D roadmaps during the endovascular procedures for both Time Period 1 and Time Period 2. The third neurointerventionalist (Group 2) performed coil embolization using the conventional 2D roadmap technique during Time Period 1. During Time Period 2, the third neurointerventionalist (Group 2) exclusively used the 3D overlay roadmap technique on 2D fluoroscopic images with commercially available software (syngoiPilot, Siemens Healthcare, Forchheim, Germany) during the endovascular procedures using only monoplane fluoroscopy. Monoplane fluoroscopy using the $3 \mathrm{D}$ overlay technique allows visualization of several working angles without the need to recreate 2D roadmap images. For stent-assisted coil embolization procedures performed for Group 2 during Time Period 2 , a $3 \mathrm{D}$ volume of the stent reconstructed from the $3 \mathrm{D}$ rotational acquisition was overlaid on 2D fluoroscopy views to enhance the visualization of stent across the aneurysm neck, minimizing coil herniation and maximizing complete aneurysm occlusion (Fig. 1). All interventional procedures were finished according to operators' discretion through confirming aneurysm occlusion on 2D DSA images.

Baseline characteristics including age, sex, location of aneurysms, aneurysm size, complex aneurysm with a wide neck $(4 \mathrm{~mm})$ or dome to neck ratio of less than 1.4 or dissection or fusiform type, and initial presentation with aneurysm rupture, anesthesia type, fluoroscopy time and radiation dose of 2D DSA, DynaCT, 3D DSA, and fluoroscopy as calculated by the angiography system were recorded. The recorded mean radiation doses indicated estimated patient exposure by vendor supplied software. Contrast volume $(\mathrm{mL})$ and procedure time (minutes) were recorded from procedure documentation. Immediate angiographic results of aneurysm occlusion by Raymond classification [9], procedure-related complications including 


\section{Neurointerventional 3D Overlay Roadmap}

thromboembolic events, access-related complications, intraprocedural aneurysm ruptures, coil migration, vessel dissection within 48 hours after intervention, and perioperative complications within 14 days including procedure-related complications, contrast-induced nephropathy with a definition of either a $25 \%$ increase in serum creatinine from baseline or $0.5 \mathrm{mg} / \mathrm{dL}$ increase in absolute value within 48-72 hours of intravenous contrast administration, postprocedural aneurysm rupture, radiation-induced effects, pneumonia, sepsis, and death were recorded. All measurements were compiled by an independent physician who did not participate in the interventional procedures.

Among 146 consecutive intracranial aneurysm patients identified, 4 patients with multiple aneurysms, 7 patients who did a protocol violation, and 4 patients with missing data were excluded. Therefore, a total of 131 aneurysms patients were included for the final analysis.

\section{D DSA acquisition and 3D overlay roadmap technique}

3D DSA datasets were acquired using a five second rotation with the following parameters: $70 \mathrm{kV}, 0.36 \mu \mathrm{Gy}$ per frame, $200^{\circ}$ rotation, $1.50^{\circ}$ angulation step per frame, and projection on $30 \times 40 \mathrm{~cm}$ flat panel detector. Reconstruction was performed on a separate workstation using manufacturer supplied software and reconstruction presets. Patient dose for each 3D DSA acquisition has been determined in a phantom study as $0.5 \mathrm{mSv}$. The 3D roadmap technique was performed according to the same manner as described by Rossitti et al (Fig. 1A) [3]. During the stent-assisted coiling, the 2D-3D coregistration method was applied that had been introduced by Richter et al (Fig. 1B) [2].

\section{Statistical Analysis}

Continuous data were reported as means \pm standard error of the mean and categorical data were reported as percentages. The Student's t-test was used to test for statistically significant differences between the means of continuous variables, and the Chi-square or Fisher's exact test was used to test for statistically significant differences between the distributions of categorical data. $P$ values were calculated as 2 -tailed and $p<0.05$ was considered statistically significant. All statistical analyses were conducted using a statistical software package (Stata 11, StataCorp, College Station, TX).
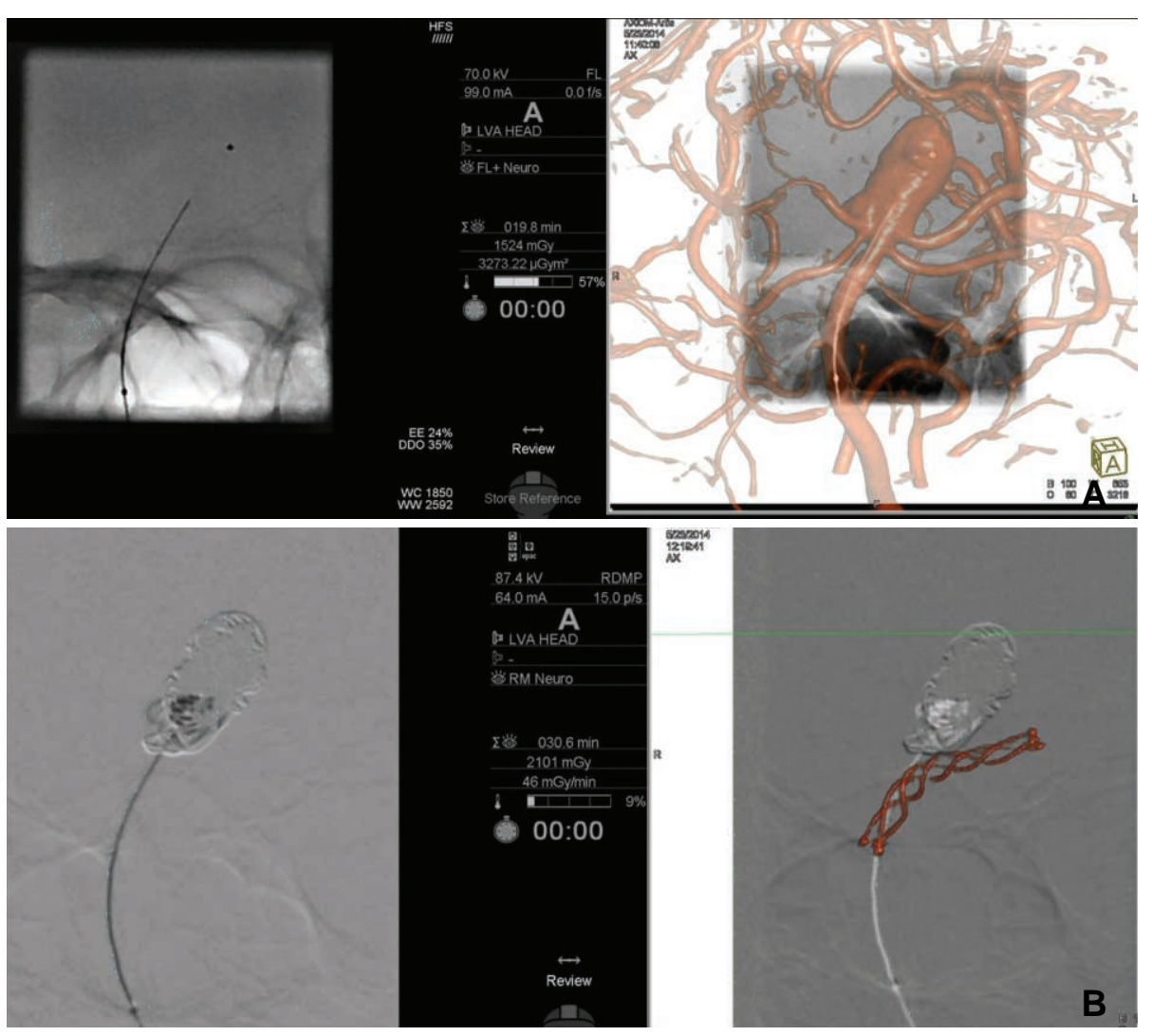

Fig. 1. Stent-assisted coil embolization of a basilar terminus aneurysm using a 3D overlay roadmap technique with monoplane fluoroscopy. After an endoluminal stent was deployed from the left posterior cerebral artery P1 segment into the distal basilar artery, a microcatheter was navigated into the aneurysm using a 3D overlay roadmap (A). Coils were deployed into the aneurysm using a blank roadmap technique and a 3D stent reconstruction overlay (B). 


\section{Dong-Kyu Jang, et al.}

\section{RESULTS}

\section{Demographics}

Patient demographics are summarized in Table 1. Among the total 131 aneurysm patients, 98 (74.8\%) patients underwent coil embolization using conventional 2D roadmap technique and $33(25.2 \%)$ patients were treated using 3D overlay roadmap technique. During Time Period 1 and Time Period 2, all baseline characteristics including age, sex, aneurysm location, aneurysm size, complex aneurysm, ruptured aneurysm, anesthesia type, and stent-assisted coiling were not significantly different between Group 1 and Group 2 $(p>0.05)$ (Table 1). However, there was significant or borderline intra-group differences regarding only two parameters of ruptured aneurysm and stent-assisted coiling of all baseline characteristics between Time
Period 1 and Time Period 2 for Group $1(\mathrm{P}=0.008$ and $\mathrm{P}<0.001)$ and Group $2(\mathrm{P}=0.074$ and $\mathrm{P}=0.013)$, respectively (Table 2). Twenty patients experienced procedural complications ; 5 thromboembolic events, 4 access-related complications, 5 contrast-induced nephropathy, 2 intraoperative rupture, 1 coil herniation, 2 postprocedural aneurysm rupture at postprocedure 1 day, 1 radiation proctitis, 1 pneumonia, and 1 arterial dissection. There were 25 patients suffered from perioperative complications including 20 patients with 23 procedural complications and 7 deaths within 14 days.

\section{Difference of Procedural Profile and Outcome Between two Groups}

During Time Period 1, there was no significant intergroup difference on radiation dose, fluoroscopy dose, fluoroscopy time, procedure time, immediate

Table 1. Intraperiod Comparison of Baseline Characteristics between Group 1 and Group 2

\begin{tabular}{|c|c|c|c|c|}
\hline Time Period & Characteristic & Group 1 & Group 2 & P Value \\
\hline \multirow[t]{9}{*}{1} & Number & 40 & 31 & \\
\hline & Age (years, mean \pm S.D.) & $55.2 \pm 14.2$ & $58.9 \pm 15.9$ & 0.30 \\
\hline & Female, n (\%) & $30(75.0)$ & $26(83.9)$ & 0.36 \\
\hline & Anterior circulation aneurysm, $\mathrm{n}(\%)$ & $34(85.0)$ & $24(77.4)$ & 0.41 \\
\hline & Aneurysm size $(\mathrm{mm})$ & $5.70 \pm 3.22$ & $5.72 \pm 3.28$ & 0.98 \\
\hline & Complex aneurysm, n (\%) & $22(55.0)$ & $19(61.3)$ & 0.6 \\
\hline & Ruptured aneurysm, n (\%) & $19(47.5)$ & $13(41.9)$ & 0.64 \\
\hline & General anesthesia, n (\%) & $34(85.0)$ & $28(90.3)$ & 0.72 \\
\hline & Stent-assisted, n (\%) & $8(20.0)$ & $11(35.5)$ & 0.14 \\
\hline \multirow[t]{9}{*}{2} & Number & 27 & 33 & \\
\hline & Age (years, mean \pm S.D.) & $58.8 \pm 10.0$ & $55.6 \pm 12.6$ & 0.29 \\
\hline & Female, n (\%) & $20(74.1)$ & $23(69.7)$ & 0.71 \\
\hline & Anterior circulation aneurysm, n (\%) & $20(74.1)$ & $28(84.8)$ & 0.30 \\
\hline & Aneurysm size (mm) & $5.89 \pm 3.26$ & $5.18 \pm 2.48$ & 0.34 \\
\hline & Complex aneurysm, n (\%) & $20(74.1)$ & $21(63.6)$ & 0.39 \\
\hline & Ruptured aneurysm, n (\%) & $4(14.8)$ & $7(21.2)$ & 0.74 \\
\hline & General anesthesia, n (\%) & $22(81.5)$ & $31(93.9)$ & 0.23 \\
\hline & Stent-assisted, n (\%) & $19(70.4)$ & $22(66.7)$ & 0.76 \\
\hline
\end{tabular}

Time Period 1: January 2012 through August 2012. Time Period 2: February 2013 through July 2013.

Group 1 includes patients that underwent coil embolization of an intracranial aneurysm by either of 2 neurointerventionalists using a conventional $2 \mathrm{D}$ roadmap technique during both time periods.

Group 2 includes patients that underwent coil embolization of an intracranial aneurysm by a third neurointerventionalist that used a conventional 2D roadmap technique during Time Period 1 and a 3D overlay roadmap technique during Time Period 2.

S.D.: standard deviation; Ave.: average; Ant. Circ.: anterior circulation and includes aneurysms at the origin of the posterior communicating artery off of the internal carotid artery. 


\section{Neurointerventional 3D Overlay Roadmap}

Table 2. Intragroup Comparison of Baseline Characteristics between Period 1 and Period 2

\begin{tabular}{|c|c|c|c|c|}
\hline Group & Characteristics & Period 1 & Period 2 & $P$ value \\
\hline \multirow[t]{8}{*}{$1(n=67)$} & Age (years, mean \pm S.D.) & $55.2 \pm 14.2$ & $58.8 \pm 10.0$ & 0.25 \\
\hline & Female, n (\%) & $30(75.0)$ & $20(74.1)$ & 0.93 \\
\hline & Anterior circulation aneurysm, $\mathrm{n}(\%)$ & $34(85.0)$ & $20(74.1)$ & 0.27 \\
\hline & Aneurysm size (mm) & $5.70 \pm 3.22$ & $5.89 \pm 3.28$ & 0.81 \\
\hline & Complex aneurysm, n (\%) & $22(55.0)$ & $20(74.1)$ & 0.11 \\
\hline & Ruptured aneurysm, n (\%) & $19(47.5)$ & $4(14.8)$ & 0.008 \\
\hline & General anesthesia, n (\%) & $34(85.0)$ & $22(81.5)$ & 0.70 \\
\hline & Stent-assisted, n (\%) & $8(20.0)$ & $19(70.4)$ & $<0.001$ \\
\hline \multirow[t]{8}{*}{$2(n=64)$} & Age (years, mean \pm S.D.) & $58.9 \pm 15.9$ & $55.6 \pm 12.6$ & 0.36 \\
\hline & Female, n (\%) & $26(83.9)$ & $23(69.7)$ & 0.18 \\
\hline & Anterior circulation aneurysm, n (\%) & $24(77.4)$ & $28(84.8)$ & 0.45 \\
\hline & Aneurysm size (mm) & $5.71 \pm 3.28$ & $5.18 \pm 2.48$ & 0.47 \\
\hline & Complex aneurysm, n (\%) & $19(61.3)$ & $21(63.6)$ & 0.85 \\
\hline & Ruptured aneurysm, n (\%) & $13(41.9)$ & $7(21.2)$ & 0.074 \\
\hline & General anesthesia, n (\%) & $28(90.3)$ & $31(93.9)$ & 0.67 \\
\hline & Stent-assisted, n (\%) & $11(35.5)$ & $22(66.7)$ & 0.013 \\
\hline
\end{tabular}

Time Period 1: January 2012 through August 2012. Time Period 2: February 2013 through July 2013.

Group 1 includes patients that underwent coil embolization of an intracranial aneurysm by either of 2 neurointerventionalists using a conventional $2 \mathrm{D}$ roadmap technique during both time periods.

Group 2 includes patients that underwent coil embolization of an intracranial aneurysm by a third neurointerventionalist that used a conventional 2D roadmap technique during Time Period 1 and a 3D overlay roadmap technique during Time Period 2. S.D.: standard deviation; Ave.: average; Ant. Circ.: anterior circulation and includes aneurysms at the origin of the posterior communicating artery off of the internal carotid artery.

angiographic occlusion, procedural complications, and perioperative complications between Group 1 and Group 2 ( $p>0.05)$; contrast administration was significantly less in Group 2 than in Group $1(\mathrm{p}=0.03)$ (Table $3)$. For Time Period 2, total radiation dose and fluoroscopy dose were significantly less in Group 2 than in Group $1(p=0.001$ and $p=0.017)$. In addition, fluoroscopy time and procedure time were significantly less in Group 2 than in Group $1(p=0.035$ and $p=0.001$ respectively). However, contrast administration, angiographic occlusion, procedural complications, and perioperative complications were not significantly different between Group 1 and Group 2 (Table 3).

\section{Intragroup Difference of Procedural Profile and Outcome between two Periods}

In a chronological analysis, Group 1 demonstrated a significant reduction of composite radiation dose of 2D DSA, DynaCT, and 3D DSA $(p=0.018)$ and contrast volume $(\mathrm{p}=0.019)$ during Time Period 2 relative to
Time Period 1 (Table 4); the other parameters were not significantly different between two Time Periods. On the other hand, Group 2 demonstrated a significant decrease of total radiation dose $(p=0.031)$, composite radiation dose of 2D DSA, DynaCT, and 3D DSA $(p<0.001)$, contrast volume $(p=0.011)$, fluoroscopy time $(p<0.001)$, procedure time $(p=0.022)$, and procedural complications $(\mathrm{p}=0.041)$ during Time Period 2 relative to Time Period 1; furthermore, the Group 2 demonstrated a trend for lower rate of perioperative complications for Time Period 2 relative to Time Period 1 ( $\mathrm{p}=0.057)$ (Table 4).

\section{DISCUSSION}

The current study demonstrated decreased fluoroscopy dose and fluoroscopy time in coil embolization procedures using a monoplane 3D overlay roadmap technique compared to a conventional biplane $2 \mathrm{D}$ roadmap technique with similar angiographic 


\section{Dong-Kyu Jang, et al.}

occlusion rates and procedure related complications.

Radiation dose reduction strategy cannot be exaggerated according to the principle of "as low as reasonably achievable" to protect the patients in case of without change of treatment outcomes. Currently, 3D DSA is primarily used to elucidate complex aneurysm morphology, select working angles, and decide potential need for a stent-assisted coil embolization procedure [5]. The 3D overlay roadmap technique enables neurointerventionists to apprehend as quickly as possible anatomical relationship during guiding catheter navigation and microcatheter selection of

Table 3. Difference of Radiation Dose, Contrast Volume, Fluoroscopy Dose, Fluoroscopy Time, Procedure Time, Angiographic Results, and Complications between Group 1 and Group 2

\begin{tabular}{|c|c|c|c|c|}
\hline Time Period & Parameter & Group 1 & Group 2 & P Value \\
\hline \multirow[t]{13}{*}{1} & Number & 40 & 31 & \\
\hline & Radiation dose (mGy) & $3381.9 \pm 271.4$ & $2790.6 \pm 299.5$ & 0.15 \\
\hline & Fluoroscopy & $2009.8 \pm 220.8$ & $1666.7 \pm 251.9$ & 0.31 \\
\hline & $2 D D S A+D y n a C T+3 D D S A$ & $1380.3 \pm 109.8$ & $1397.5 \pm 129.2$ & 0.92 \\
\hline & Contrast (mL) & $168.5 \pm 9.8$ & $141.3 \pm 7.3$ & 0.03 \\
\hline & Fluoroscopy time (min) & $60.7 \pm 5.5$ & $53.3 \pm 4.7$ & 0.33 \\
\hline & Procedure time (min) & $105.3 \pm 8.5$ & $107.1 \pm 8.8$ & 0.89 \\
\hline & Raymond class, n (\%) & & & 0.84 \\
\hline & 1 & $20(50.0)$ & $17(54.8)$ & \\
\hline & 2 & $14(35.0)$ & $11(35.5)$ & \\
\hline & 3 & $6(15.0)$ & $3(9.7 \%)$ & \\
\hline & Procedural complications, n (\%) & $6(15.0)$ & $8(25.8)$ & 0.26 \\
\hline & Perioperative complications, $\mathrm{n}(\%)$ & $9(22.5)$ & $9(29.0)$ & 0.53 \\
\hline \multirow[t]{13}{*}{2} & Number & 27 & 33 & \\
\hline & Radiation dose (mGy) & $3375.0 \pm 314.3$ & $1953.4 \pm 238.3$ & 0.001 \\
\hline & Fluoroscopy & $1998.5 \pm 228.0$ & $1262.2 \pm 195.6$ & 0.017 \\
\hline & $2 D D S A+D y n a C T+3 D D S A$ & $1063.9 \pm 69.0$ & $710.1 \pm 75.4$ & 0.001 \\
\hline & Contrast (mL) & $133.0 \pm 10.5$ & $112.0 \pm 8.4$ & 0.12 \\
\hline & Fluoroscopy time (min) & $62.3 \pm 6.2$ & $30.5 \pm 3.6$ & $<0.001$ \\
\hline & Procedure time (min) & $105.0 \pm 7.8$ & $82.2 \pm 6.1$ & 0.023 \\
\hline & Raymond class, n (\%) & & & 0.14 \\
\hline & 1 & $9(33.3)$ & $19(57.6)$ & \\
\hline & 2 & $10(37.0)$ & $6(18.2)$ & \\
\hline & 3 & $8(29.6)$ & $8(24.2)$ & \\
\hline & Procedural complications, n (\%) & $4(14.8)$ & $2(6.1)$ & 0.39 \\
\hline & Perioperative complications, n (\%) & $4(14.8)$ & $3(9.1)$ & 0.49 \\
\hline
\end{tabular}

Time Period 1: January 2012 through August 2012. Time Period 2: February 2013 through July 2013.

Group 1 includes patients that underwent coil embolization of an intracranial aneurysm by either of 2 neurointerventionalists using a conventional 2D roadmap technique during both time periods.

Group 2 includes patients that underwent coil embolization of an intracranial aneurysm by a third neurointerventionalist that used a conventional 2D roadmap technique during Time Period 1 and a 3D overlay roadmap technique during Time Period 2.

Values reported at the mean \pm the standard error of the mean. 3D DSA and min mean three dimensional rotational angiography and minute, respectively. 


\section{Neurointerventional 3D Overlay Roadmap}

target aneurysms. Also as illustrated by the case example (Fig. 1), a 3D reconstruction of an intracranial stent overlaid on 2D fluoroscopy can facilitate coil delivery through a deployed stent. Nevertheless, total procedure time in the group using a 2D roadmap technique was shorter relative to a $3 \mathrm{D}$ overlay roadmap for uterine artery catheterizations in a prospective, randomized study, though there was no difference for radiation exposure or contrast load between the two techniques [4]. A transition period from the 2D roadmap to the $3 \mathrm{D}$ overlay roadmap technique may be required to master the newer 3D technique [4]. On the contrary, after allowing a transition period for a neurointerventionist to master the $3 \mathrm{D}$ overlay roadmap technique in the current study, Group 2 demonstrated significant less amount of all radiation dose measures and shorter fluoroscopy time and procedure time relative to Group 1 during Time Period 2 (Table 3).

Table 4. Difference of Radiation Dose, Contrast Volume, Fluoroscopy Dose, Fluoroscopy Time, Procedure Time, Angiographic Results, and Complications between Period 1 and Period 2

\begin{tabular}{|c|c|c|c|c|}
\hline Group & Characteristics & Period 1 & Period 2 & $P$ value \\
\hline \multirow[t]{12}{*}{$1(n=67)$} & Radiation dose (mGy) & $3381.9 \pm 271.4$ & $3375.0 \pm 314.3$ & 0.99 \\
\hline & Fluoroscopy & $2009.8 \pm 220.8$ & $1998.5 \pm 228.0$ & 0.97 \\
\hline & $2 D D S A+D y n a C T+3 D D S A$ & $1380.3 \pm 109.8$ & $1063.9 \pm 69.0$ & 0.018 \\
\hline & Contrast $(\mathrm{mL})$ & $168.5 \pm 9.8$ & $133.0 \pm 10.5$ & 0.019 \\
\hline & Fluoroscopy time (min) & $60.7 \pm 5.5$ & $62.3 \pm 6.2$ & 0.85 \\
\hline & Procedure time (min) & $105.3 \pm 8.5$ & $105.0 \pm 7.8$ & 0.98 \\
\hline & Raymond class, n (\%) & & & 0.26 \\
\hline & 1 & $20(50.0)$ & $9(33.3)$ & \\
\hline & 2 & $14(35.0)$ & $10(37.0)$ & \\
\hline & 3 & $6(15.0)$ & $8(29.6)$ & \\
\hline & Procedural complications, $\mathrm{n}(\%)$ & $6(15.0)$ & $4(14.8)$ & 1.0 \\
\hline & Perioperative complications, n (\%) & $9(22.5)$ & $4(14.8)$ & 0.54 \\
\hline \multirow[t]{12}{*}{$2(n=64)$} & Radiation dose (mGy) & $2790.6 \pm 299.5$ & $1953.4 \pm 238.3$ & 0.031 \\
\hline & Fluoroscopy & $1666.7 \pm 251.9$ & $1262.2 \pm 195.6$ & 0.21 \\
\hline & $2 D D S A+D y n a C T+3 D D S A$ & $1397.5 \pm 129.2$ & $710.1 \pm 75.4$ & $<0.001$ \\
\hline & Contrast $(\mathrm{mL})$ & $141.3 \pm 7.3$ & $112.0 \pm 8.4$ & 0.011 \\
\hline & Fluoroscopy time (min) & $53.3 \pm 4.7$ & $30.5 \pm 3.6$ & $<0.001$ \\
\hline & Procedure time (min) & $107.1 \pm 8.8$ & $82.2 \pm 6.1$ & 0.022 \\
\hline & Raymond class, n (\%) & & & 0.16 \\
\hline & 1 & $17(54.8)$ & $19(57.6)$ & \\
\hline & 2 & $11(35.5)$ & $6(18.2)$ & \\
\hline & 3 & $3(9.7)$ & $8(24.2)$ & \\
\hline & Procedural complications, n (\%) & $8(25.8)$ & $2(6.1)$ & 0.041 \\
\hline & Perioperative complications, $\mathrm{n}(\%)$ & $9(29.0)$ & $3(9.1)$ & 0.057 \\
\hline
\end{tabular}

Time Period 1: January 2012 through August 2012. Time Period 2: February 2013 through July 2013.

Group 1 includes patients that underwent coil embolization of an intracranial aneurysm by either of 2 neurointerventionalists using a conventional 2D roadmap technique during both time periods.

Group 2 includes patients that underwent coil embolization of an intracranial aneurysm by a third neurointerventionalist that used a conventional 2D roadmap technique during Time Period 1 and a 3D overlay roadmap technique during Time Period 2.

Values reported at the mean \pm the standard error of the mean. 3D DSA and min mean three dimensional rotational angiography and minute, respectively. 


\section{Dong-Kyu Jang, et al.}

Group 1, which continued to employ the conventional 2D roadmap technique throughout the study period, demonstrated significant reduction of composite radiation dose of 2D DSA and DynaCT and 3D DSA from Time Period 1 to Time Period 2 (Table 4). In addition, Group 2 showed significant reductions of total radiation dose, composite radiation dose of $2 \mathrm{D}$ DSA and DynaCT and 3D DSA, and contrast volume from Time Period 1 to Time Period 2 (Table 4). In addition, fluoroscopy time and procedure time were significantly reduced in Group 2 from Time Period 1 to Time Period 2. Procedure complications of Group 2 were less in Time Period 2 than in Time Period 1 without difference of angiographic results (Table 4). Therefore, our results suggest that 3D overlay roadmap technique can reduce total radiation dose and fluoroscopy dose.

Regarding contrast injection, compared to Time Period 1, there was less administration of contrast volume in both of Group 1 and Group 2 during Time Period 2 (Table 4); moreover, Group 1 and Group 2 showed less composite radiation dose of 2D DSA and DynaCT and 3D DSA in Time Period 2 than in Time Period 1 (Table 4). Consequently, contrast volume reduction may be associated with acquisition of the other images except for fluoroscopy images. The expected continued refinements of angiography technique and different samples of population between Time Period 1 and Time Period 2 are at least partially accounted for less administration of contrast volume.

The 3D overlay roadmap technique allows an interventionist to readily apprehend 3D anatomy of vessels and freely change between various working angles without the need to obtain new 2D roadmaps, reducing additional radiation and contrast dose. These reductions in turn may reduce perioperative complications such as thromboembolisms and contrast induced nephrotoxicity [10-12]. In our study, less fluoroscopy time and procedure time in Time Period 2 than Time Period 1 of Group 2 was revealed. During Time Period 2 , there was no differentiation of complications rates between Group 1 and Group 2 (Table 3) and there was significant reduction of procedural complications of Group 2 during Time Period 2 than during Time Period 1 in spite of non blindness of neurointerventionists for roadmap techinuqes (Table 4).

As mentioned by Rossitti et al [3], 3D overlay roadmap technique is not "True $3 \mathrm{D}$ road map technique" but "intermediate 3D roadmap technique" because 3D overlay roadmap technique does not use a 3D monitor. In addition, there are still several doubts whether monoplane 3D overlay roadmap technique can substitute for biplane angiography system during endovascular procedures of cerebral aneurysms. In this study, Group 2 included a single neurointerventionist. The positive procedural effects of monoplane 3D roadmap technique might be attributed to experience gained by the Group 2 over the course of time. Lastly, $3 \mathrm{D}$ overlay roadmap technique can not adjust immediately the change in vascular anatomy that occurs with the placement of microcatheters and intravascular implants. Even so, monoplane 3D overlay roadmap technique enabled neurointerventionists to perform embolization with shorter procedure time and less radiation dose using monoplane angiography system. This implies the possibility of compensation of limitations of monoplane angiography system and further gives us a hint to improve procedural effects during the interventions using biplane angiography suites.

The retrospective nature and lack of randomization are limitations of the current study. Although factors such as experience and practice preferences among the neurointerventionists may have confounded results, the intervention skill among the interventionists was assumed to be equivalent throughout the study period. While the 3D overlay roadmap technique is currently only available for the primary plane of a Biplane system, a natural extension of it to the second plane could potentially further improve the endovascular procedure guidance. Such an extension could further shorten procedure and fluoroscopic time, by allowing a second working angle, alleviating the need for $\mathrm{C}$-arm movement, minimizing workflow interruptions.

\section{CONCLUSION}

The 3D overlay roadmap technique for endovascular cerebral aneurysm coil embolization procedures using monoplane fluoroscopy can successfully fluoroscopy dose and fluoroscopy time relative to using a conventional 2D roadmap technique using biplane fluoroscopy. With these findings, 3D overlay roadmap technique is another tool in a physician's arsenal to keep patient radiation exposure as low as reasonably achievable and may ultimately improve procedure efficiency and possibly patients' outcomes as the technology improves. This may give a hint of compensation of limitations of monoplane angiography system to us during neuroendovascular procedures.

\section{Acknowledgement}

We would like to recognize Meghana Shownkeen for 


\section{Neurointerventional 3D Overlay Roadmap}

her contributions throughout this project.

\section{Disclosure}

Dong-Kyu Jang, David A. Stidd, Michael Chen, and Roham Moftakhar report no conflict of interest concerning the materials or methods used in this study. Sebastian Schafer is an employee of Siemens Medical Solutions, USA. Demetrius K. Lopes has received research grants from Medtronic, Microvention, Siemens Medical Solutions, Stryker, and Penumbra, and is on the advisory board of Medtronic, Stryker, Penumbra, Blockade, and Siemens Medical Solutions.

\section{References}

1. Anxionnat R, Bracard S, Ducrocq X, Trousset Y, Launay L, Kerrien E, et al. Intracranial aneurysms: clinical value of $3 \mathrm{D}$ digital subtraction angiography in the therapeutic decision and endovascular treatment. Radiology 2001;218:799-808

2. Richter G, Pfister M, Struffert T, Engelhorn T, Doelken M, Spiegel M, et al. Technical feasibility of 2D-3D coregistration for visualization of self-expandable microstents to facilitate coil embolization of broad-based intracranial aneurysms: an in vitro study. Neuroradiology 2009;51:851-854

3. Rossitti S, Pfister M. 3D Road-Mapping in the Endovascular Treatment of Cerebral Aneurysms and Arteriovenous Malformations. Interv Neuroradiol 2009;15:283-290
4. Maleux G, Michielsen K, Timmerman D, Poppe W, Heye S, Vaninbroukx J, et al. 2D versus 3D roadmap for uterine artery catheterization: impact on several angiographic parameters. Acta Radiol 2014;55:62-70

5. Loh Y, McArthur DL, Tateshima S, Jahan R, Duckwiler GR, Vinuela F. Safety of intracranial endovascular aneurysm therapy using 3-dimensional rotational angiography: a single-center experience. Surg Neurol 2008;69:158-163; discussion 163

6. Soderman M, Babic D, Homan R, Andersson T. 3D roadmap in neuroangiography: technique and clinical interest. Neuroradiology 2005;47:735-740

7. Bakker, den Heeten, van Rooij, Grimbergen. Image guidance for neurovascular intervention: proposed setup for a 3D-roadmap system. Minim Invasive Ther Allied Technol 2003;12:304-309

8. Johnson, Coley SJ, Kyrion J, Taylor WJ. Comparing the performance of mono- and biplane fluoroscopy systems in diagnostic and interventional neuroangiography using the dose-area product. Neuroradiology 2001;43:728-734

9. Roy D, Milot G, Raymond J. Endovascular treatment of unruptured aneurysms. Stroke 2001;32:1998-2004

10. Wong JM, Ziewacz JE, Panchmatia JR, Bader AM, Pandey AS, Thompson BG, et al. Patterns in neurosurgical adverse events: endovascular neurosurgery. Neurosurg Focus 2012;33:E14

11. Bakker NH, Tanase D, Reekers JA, Grimbergen CA. Evaluation of vascular and interventional procedures with time-action analysis: a pilot study. J Vasc Interv Radiol 2002;13:483-488

12. Prasad V, Gandhi D, Stokum C, Miller T, Jindal G. Incidence of contrast material-induced nephropathy after neuroendovascular procedures. Radiology 2014;273:853-858 